OPEN ACCESS

Edited by:

Mats Bemark,

University of Gothenburg, Sweden

Reviewed by:

Charles Kelly,

King's College London,

United Kingdom

Hyun-Dong Chang,

Deutsches Rheuma-

Forschungszentrum (DRFZ),

Germany

*Correspondence: Gihyun Lee

glee@khu.ac.kr

Hyunsu Bae

hbae@khu.ac.kr

tThese authors have contributed equally to this work

Specialty section:

This article was submitted to

Mucosal Immunity,

a section of the journal

Frontiers in Immunology

Received: 17 April 2018

Accepted: 27 February 2019

Published: 21 March 2019

Citation:

Jung K-H, Shin D, Kim S, Min D,

Kim W, Kim J, Lee G and Bae H

(2019) Intratracheal Ovalbumin

Administration Induces Colitis Through

the IFN- $\gamma$ Pathway in Mice.

Front. Immunol. 10:530.

doi: 10.3389/fimmu.2019.00530

\section{Intratracheal Ovalbumin Administration Induces Colitis Through the IFN- $\gamma$ Pathway in Mice}

\author{
Kyoung-Hwa Jung ${ }^{1 \dagger}$, Dasom Shin ${ }^{1+}$, Sejun Kim ${ }^{1}$, Daeun Min ${ }^{1}$, Woogyeong Kim ${ }^{2}$, \\ Jinju Kim², Gihyun Lee ${ }^{3 *}$ and Hyunsu Bae ${ }^{1 *}$
}

${ }^{1}$ Department of Physiology, College of Korean Medicine, Kyung Hee University, Seoul, South Korea, ${ }^{2}$ Department of Korean Physiology, College of Pharmacy, Kyung Hee University, Seoul, South Korea, ${ }^{3}$ College of Korean Medicine, Dongshin University, Naju, South Korea

Recent studies have reported an increased incidence of inflammatory bowel disease (IBD) in patients with pulmonary diseases. Despite clinical and epidemiological studies of the interplay between colitis and asthma, the diseases' related underlying mechanisms remain unclear. In this study, we evaluated the development of colitis in a model of allergic airway inflammation. We revealed that intratracheal chronic ovalbumin (OVA) exposure induces colitis and allergic airway inflammation. Interestingly, induction of colitis was largely regulated by Th1, rather than Th2 responses, whereas allergic airway inflammation was primarily mediated by Th2 responses. Experiments in Tbx21 (T-bet) and Ifng (IFN- $\gamma$ ) deficient mice have confirmed that IFN- $\gamma$ is a major mediator involved in OVA-induced colitis. These findings broaden current understanding of allergen induced colitis pathology and could play a role in the development of novel clinical treatment strategies for asthmatic patients who are at risk of developing colitis.

Keywords: allergy and immunology, colitis, IFN- $\gamma$, ovalbumin, Th1 cells

\section{INTRODUCTION}

Cytokines play a crucial role in the pathogenesis of inflammatory bowel diseases (IBD), such as Crohn's disease, regulating diverse aspects of the inflammatory reaction (1). IFN- $\gamma$ is a primary proinflammatory cytokine involved in Crohn's disease pathogenesis (2). IFN- $\gamma$ is critical in the regulation of multiple immune functions, such as antigen presentation, cellular proliferation, leukocyte trafficking, microbicide effector activation, and pathogen recognition (3). However, overproduction of IFN- $\gamma$ is implicated in many gastrointestinal disorders, including Crohn's disease, celiac disease, and autoimmune gastritis (4-6), and also plays a role in pulmonary inflammation (7).

Several recent studies have reported a link between inflammatory bowel disease and pulmonary inflammation (8-11) Incidence of IBD is significantly increased among patients with lung disorders (12). Despite these epidemiological and clinical observations, few experimental studies have investigated the role of airway allergic responses in the development of IBD. To reveal the mechanisms underlying the effects of allergic airway inflammation on IBD pathogenesis, we hypothesized that intratracheal ovalbumin (OVA) exposure would induce inflammation in both the lung and the colon, as it is well-known that OVA exposure causes Th2- and Th1-mediated airway inflammation responses (13). Notably, we observed that intratracheal OVA exposure is sufficient to induce colitis mediated by Th1 responses, not Th2 responses. Based on these results, we investigated 
the role of Th1 responses in OVA exposure induced colitis using T-bet or IFN- $\gamma$ deficient mice. In this study, we demonstrate that IFN- $\gamma$ plays a key role in colitis induced by intratracheal exposure to OVA.

\section{MATERIALS AND METHODS}

\section{Animals}

This study was approved by the Kyung Hee University animal care and use committee. All of the experiments were performed in accordance with the approved animal protocols and guidelines established by Kyung Hee University [KHUASP(SE)17-039]. Female 6- to 8-week-old C57BL/6 mice were used for experiments. Ancestors of WT and IFN- $\gamma$ knockout (Ifng ${ }^{-/-}$, B6.129S7-Ifngtm1Ts/J) mice used for this study were purchased from the Jackson Laboratory (Bar Harbor, ME, USA). Ancestor of T-bet knockout (Tbet ${ }^{-/}$) mice were provided by Dr. Laurie Glimcher (Dana-Farber Cancer Institute). All mice have been bred in the same room for at least 10 generations, eating the same food to exclude the influence of microbiota. Sex-matched littermate controls were used in all experiments. All of the mice were housed under specific pathogen-free conditions in the Animal Barrier Facility.

\section{Animal Models of Allergic Airway Disease and Colitis}

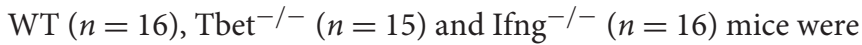
sensitized on days 0 and 14 by intraperitoneal (i.p.) injection of $5 \mathrm{mg}$ of OVA (Sigma-Aldrich) emulsified in $1 \mathrm{mg}$ of aluminum hydroxide. Subsequently, sensitized mice were intratracheally applied with $0.5 \mu \mathrm{g}$ of OVA in $50 \mu \mathrm{l}$ of PBS on days 22, 23, 24, 29, 30, and 31 after initial OVA exposure (Figure 1A). Twenty-four $\mathrm{h}$ after the last challenge, mice were sacrificed $(14,15)$.

\section{Cytokine Measurement}

Colonic tissues and lung tissues were homogenized with a T-PER tissue lysis buffer (Thermo Fisher Scientific Inc., Waltham, MA, USA) containing a protease inhibitor cocktail (Roche Diagnostics, Mannheim, Germany) using a Precellys 24 homogenizer (Bertin Technologies, France) and centrifuged at $13,000 \mathrm{rpm}$ for $15 \mathrm{~min}$ at $4^{\circ} \mathrm{C}$. Colon cytokine levels in the homogenates were measured using a murine Th1, 2 and 17 Cytometric Bead Array (CBA kit; BD sciences, San Diego, CA, USA) or ELISA kits [IL-4, IL-5, IL-6, IFN- $\gamma$, TNF- $\alpha$ from BD sciences (16); IL-2, IL-13, IL-17, IL-22 from R\&D, Minneapolis, MN, USA (17)]. Lung cytokine levels in the homogenates were measured using ELISA kits [IL-4, IL-5, IL-6, IFN- $\gamma$, TNF- $\alpha$ from BD sciences (16); IL-13 from R\&D, Minneapolis, MN, USA (17)]. Total protein concentrations were characterized with a Bio-Rad protein assay (BioRad, Hercules, CA, USA) read on a microplate reader (SOFT max PRO software, Sunnyvale, CA, USA).

\section{Histological Analysis}

Longitudinally divided rolled-up parts of the colon were used for histological analysis. Harvested lung tissues were directly fixed in $10 \%$ neutral-buffered formalin overnight at $4^{\circ} \mathrm{C}$. The tissue samples were dehydrated and then paraffin-embedded (cut into colon: $10-\mu \mathrm{m}$-thick; lung: $4-\mu \mathrm{m}$-thick sections) using a rotary microtome. The four sections of colon and lung were stained with hematoxylin \& eosin $(\mathrm{H} \& \mathrm{E})$, respectively. Image of colon and lung sections were captured using an Olympus BX51 microscope (Olympus, Tokyo, Japan) equipped with a DP71 digital camera (Olympus, Tokyo, Japan) under x200 magnifications. Colon histological samples were scored by blinded investigators as described by Erben et al. (18). Colon samples were scored between 0 and 5, with the score calculated using the following criteria: $0=$ normal colon mucosa with intact epithelium; $1=$ scattered inflammatory cell infiltrates in the mucosa; $2=$ diffuse mucosal infiltrates without submucosal spreading and an intact epithelial layer; $3=$ moderate infiltration of inflammatory cells into the mucosa and submucosa with epithelial hyperplasia and goblet cell loss; $4=$ marked infiltration of inflammatory cells into the mucosa and submucosa accompanied by crypt abscesses and loss of goblet cells and crypts; $5=$ marked infiltration of inflammatory cells into the mucosa and submucosa accompanied by crypt loss and hemorrhage. Lung samples were scored as: $0=$ normal; $1=$ very mild; $2=$ mild; $3=$ moderate; $4=$ marked; or $5=$ severe inflammation as described by Tate et al. (19).

\section{Differential (Diff) Cells Counts in BAL Fluid}

To collect BAL fluid, ice-cold PBS $(1 \mathrm{ml})$ was infused into the lungs and withdrawn via tracheal cannulation three times, respectively (harvested BAL fluid volume $2.0-2.5 \mathrm{ml}$ ). The harvested BAL fluid was centrifuged at $1,300 \mathrm{rpm}$ for $10 \mathrm{~min}$ at $4{ }^{\circ} \mathrm{C}$; supernatants were removed and the BAL cellular pellet was re-suspended in $1 \mathrm{ml}$ of ice-cold PBS. Next, BAL cells were adhered to glass sides using a Cytospin (Sandon, Waltham, MA, USA) and stained with Diff-Quick. The stained BAL cells were dried and mounted with a non-aqueous medium (Diamount, Diapath. Martinengo, BG, Italy). The BAL cells were counted under a light microscope following the method we previously reported (15).

\section{FTY720 Treatment and $\alpha 4 \beta 7$ Measurement}

WT mice $(n=16)$ were sensitized on days 0 and 14 by i.p. injection of OVA (Sigma-Aldrich; $5 \mathrm{mg}$ ) emulsified in aluminum hydroxide (1 mg). FTY720 (Sigma-Aldrich, St. Louis, Missouri, USA; $0.8 \mathrm{mg} / \mathrm{kg}$ ) was administered intraperitoneally $30 \mathrm{~min}$ prior to intratracheal OVA administration on days 22, 23, 24, 39,30 , and $31(20,21)$. The spleens were disrupted over a wire mesh screen in $2 \mathrm{ml}$ PBS. The spleen supernatant was collected in a $15 \mathrm{ml}$ tube and centrifuged for $10 \mathrm{~min}$ at $300 \mathrm{~g}$. The supernatant was removed and BD Pharm Lyse ${ }^{\text {TM }}$ lysing solution (BD sciences, San Diego, CA, USA) added to the red blood cells for $5 \mathrm{~min}$. The cells were washed with $9 \mathrm{ml}$ PBS and centrifuged for $10 \mathrm{~min}$ at $300 \mathrm{~g}$ to give a single cell suspension ready for staining. To measure gut homing integrin $\alpha 4 \beta 7$, splenocytes were incubated with CD4-APC and $\alpha 4 \beta 7$-PE antibodies (e-Bioscience, San Diego, CA, USA). Samples were first gated for lymphocytes, and then for CD4 positive cells; finally, to analyze $\alpha 4 \beta 7$ in CD 4 cells, CD 4 positive and $\alpha 4 \beta 7$ positive cells were gated. All sample data were acquired using FACSCalibur (BD sciences, San Diego, CA, USA) and analyzed using FlowJo software (Tree Star Inc., Ashland, OR, USA). 


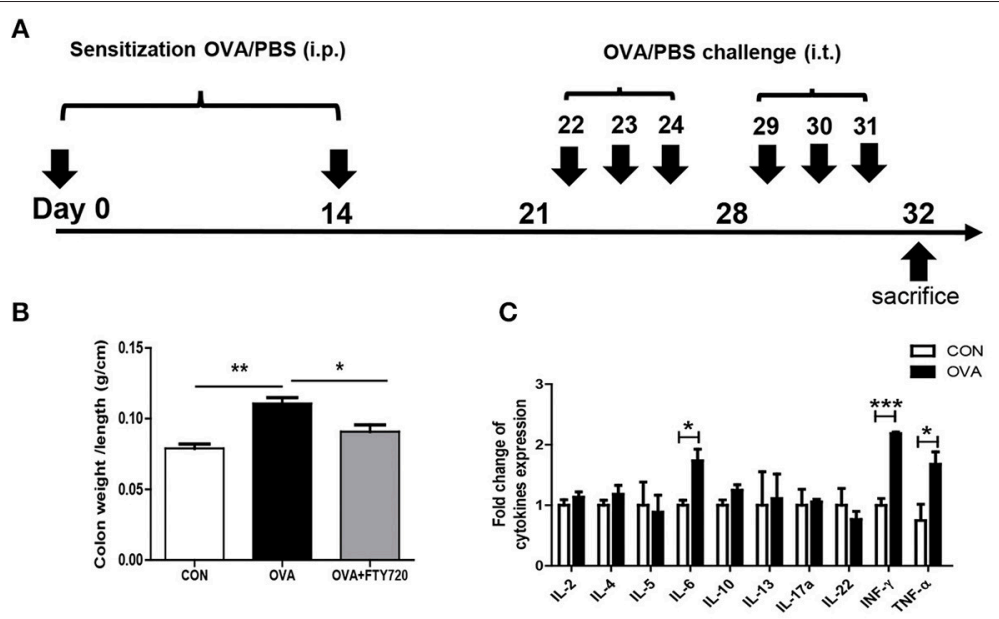

C
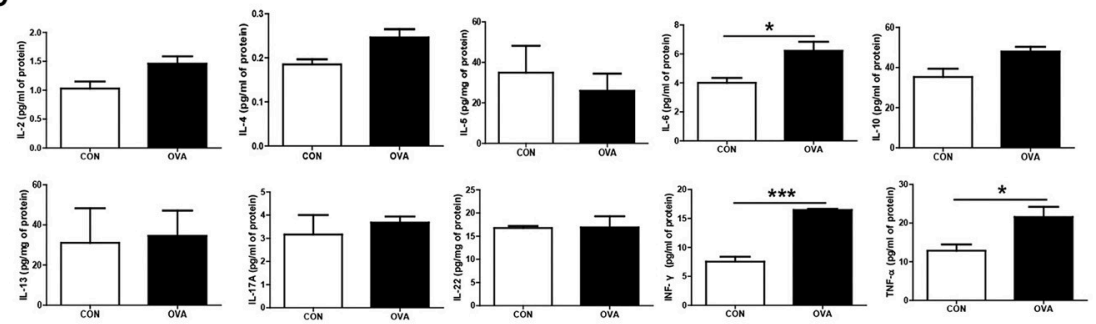

E
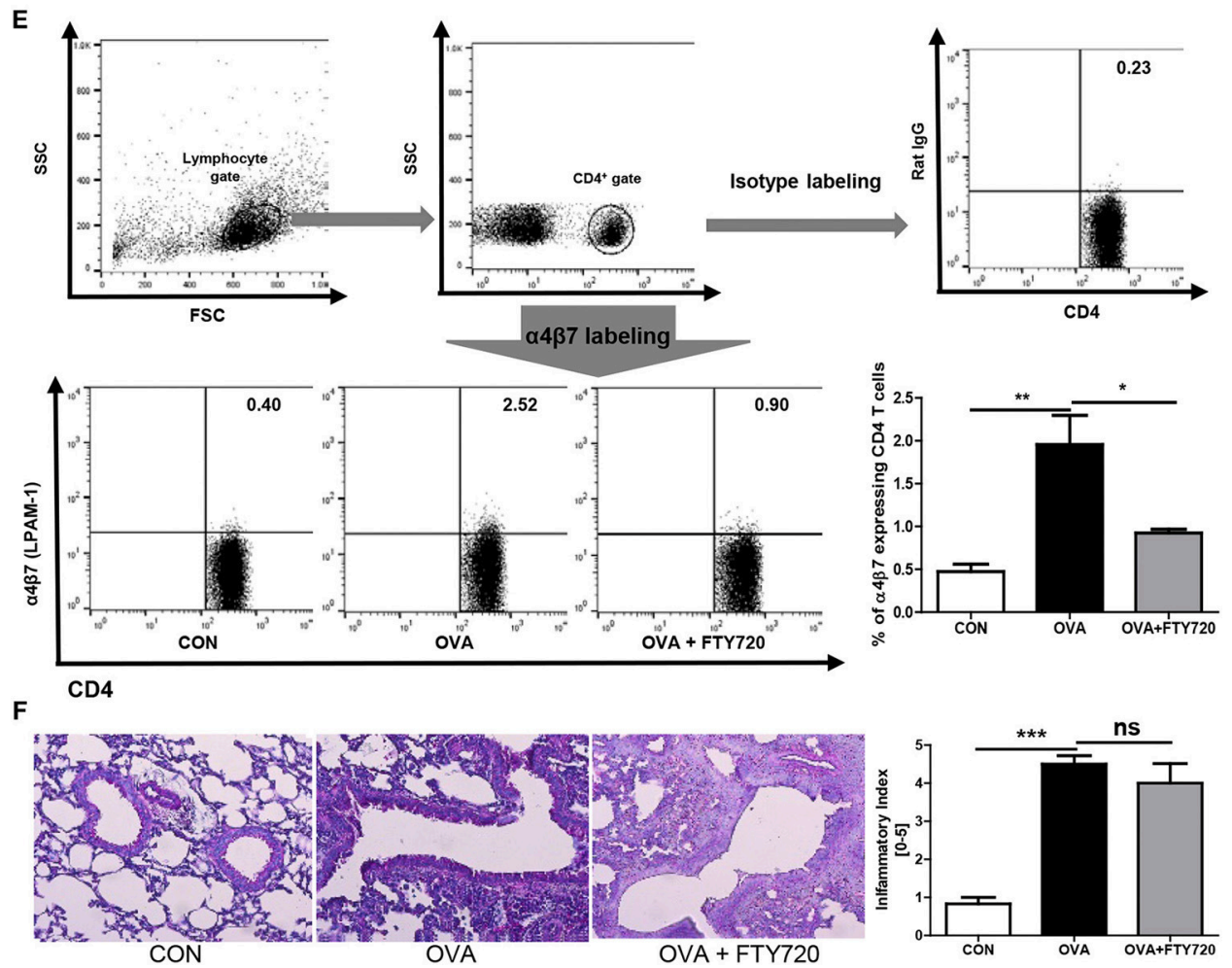

FIGURE 1 | Induction of OVA allergen related colitis. (A) Mice $(n=16)$ were sensitized via i.p. injection of OVA mixed with aluminum hydroxide on days 0 and 14. Sensitized mice were intratracheally challenged with 1\% OVA, six times between days 22 and 31. Normal control (CON) mice were sensitized and challenged with PBS alone. (B) The ration between the colon weight and length are shown. (C,D) The expression of cytokines in the colon after OVA exposure is shown. (E) The percentage of $\alpha 4 \beta 7$ expressing CD4 cells are shown. (F) A representative image of lung histology after OVA and FTY720 treatment and quantified score of severity of allergic airway inflammation are shown. Data are presented as the mean \pm SEM and the $p$-value was estimated by unpaired $t$-test $\left({ }^{\star} P<0.05\right.$, ${ }^{\star \star} P<0.01$, and $\left.{ }^{\star \star *} P<0.001\right)$. 


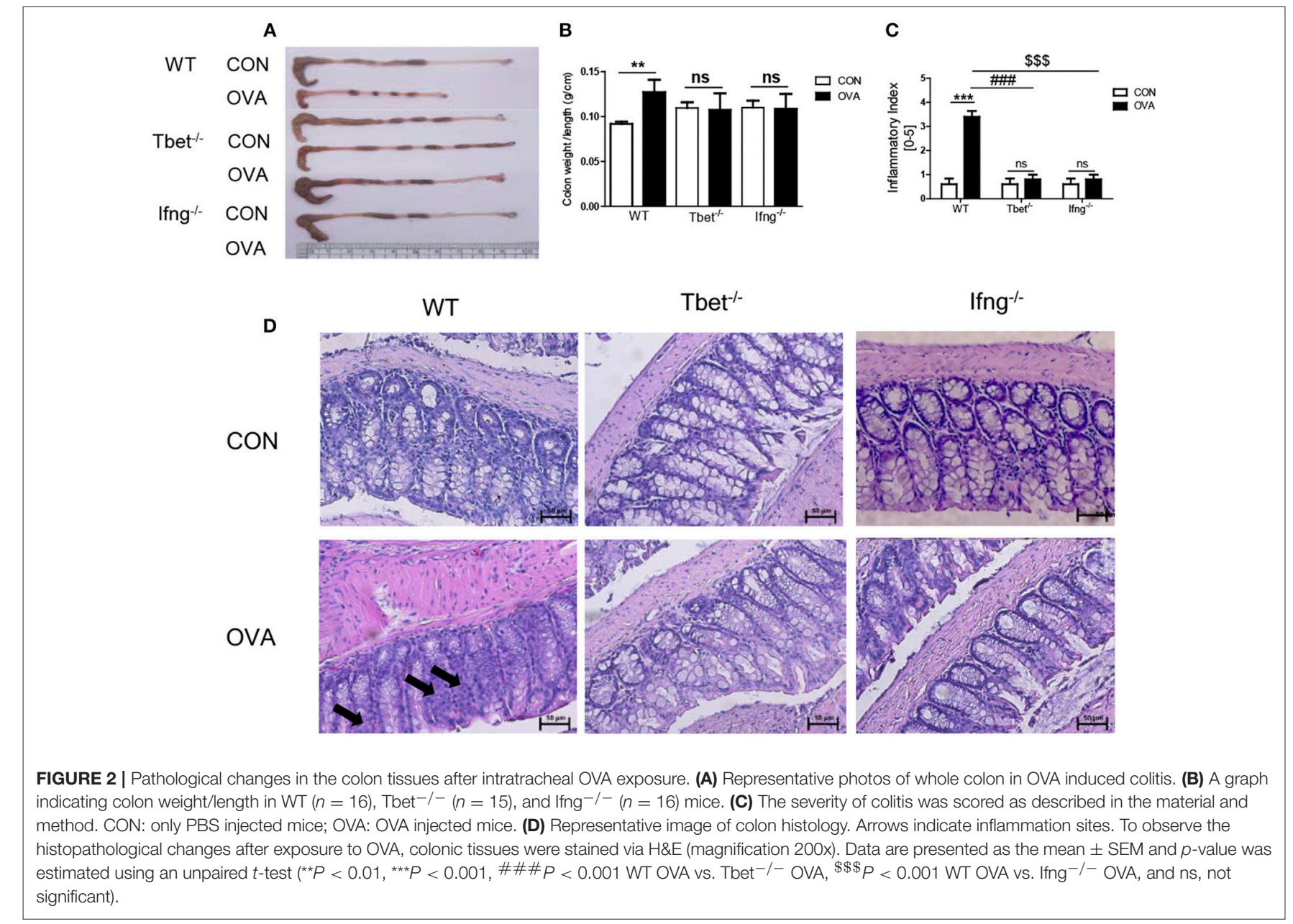

\section{Isolation of Colonic Lamina Propria Leukocytes}

To isolate the colonic lamina propria, entire colons were cut longitudinally and washed to remove feces and debris. Sections of colon were incubated in HBSS containing $5 \mathrm{Mm}$ EDTA and $2 \%$ FBS in a shaking incubator at $37^{\circ} \mathrm{C}$ for $25 \mathrm{~min}$. After washing three times in PBS to remove EDTA, the colon sections were finely minced and incubated in digestion media containing HBSS, $1 \mathrm{mg} / \mathrm{mL}$ collagenase VIII (Sigma-Aldrich), $0.01 \mathrm{mg} / \mathrm{mL}$ DNase (Roche), and $1 \mathrm{M} 2 \beta \mathrm{ME}$ (Sigma-Aldrich) in a shaking incubator at $37^{\circ} \mathrm{C}$ for $45 \mathrm{~min}$. Following collagenase digestion, the medium containing the mononuclear cells was collected, filtered, and centrifuged at $300 \mathrm{~g}$ for $10 \mathrm{~min}$.

\section{Statistical Analysis}

The statistical analyses of the data were conducted using Prism 5 software (GraphPad Software Inc., La Jolla, CA, USA). Date represented as the means \pm SEM. The statistical significance $(p<0.05)$ was assessed using an unpaired $t$-test.

\section{RESULTS \\ Chronic Intratracheal OVA Exposure Induces Colitis}

Chronic OVA exposure is commonly used to establish allergic airway inflammation in mice. To determine if chronic OVA exposure causes development of colitis, we challenged mice with PBS or OVA following an established protocol for a murine model of asthma (Figure 1A).

As shown in Figure 1B, colonic weight/length was significantly increased in the OVA group (OVA challenged mice) compared to the control group (PBS challenged mice). To elucidate the immunological mechanisms of allergic airway inflammation-induced colitis, we analyzed cytokine expression in colonic tissue. Surprisingly, levels of IFN- $\gamma$ were highly elevated; other proinflammatory cytokines were elevated, including TNF- $\alpha$ and IL-6, suggesting that OVA-induced colitis is mediated through Th1 responses. Interestingly, there was no increase in the level of cytokines, including IL-4, IL-5, IL-13, IL-17, and IL-22, produced by Th2 and Th17 cells in the colon (Figures 1C,D), but there was in the lungs (Figure 4). 

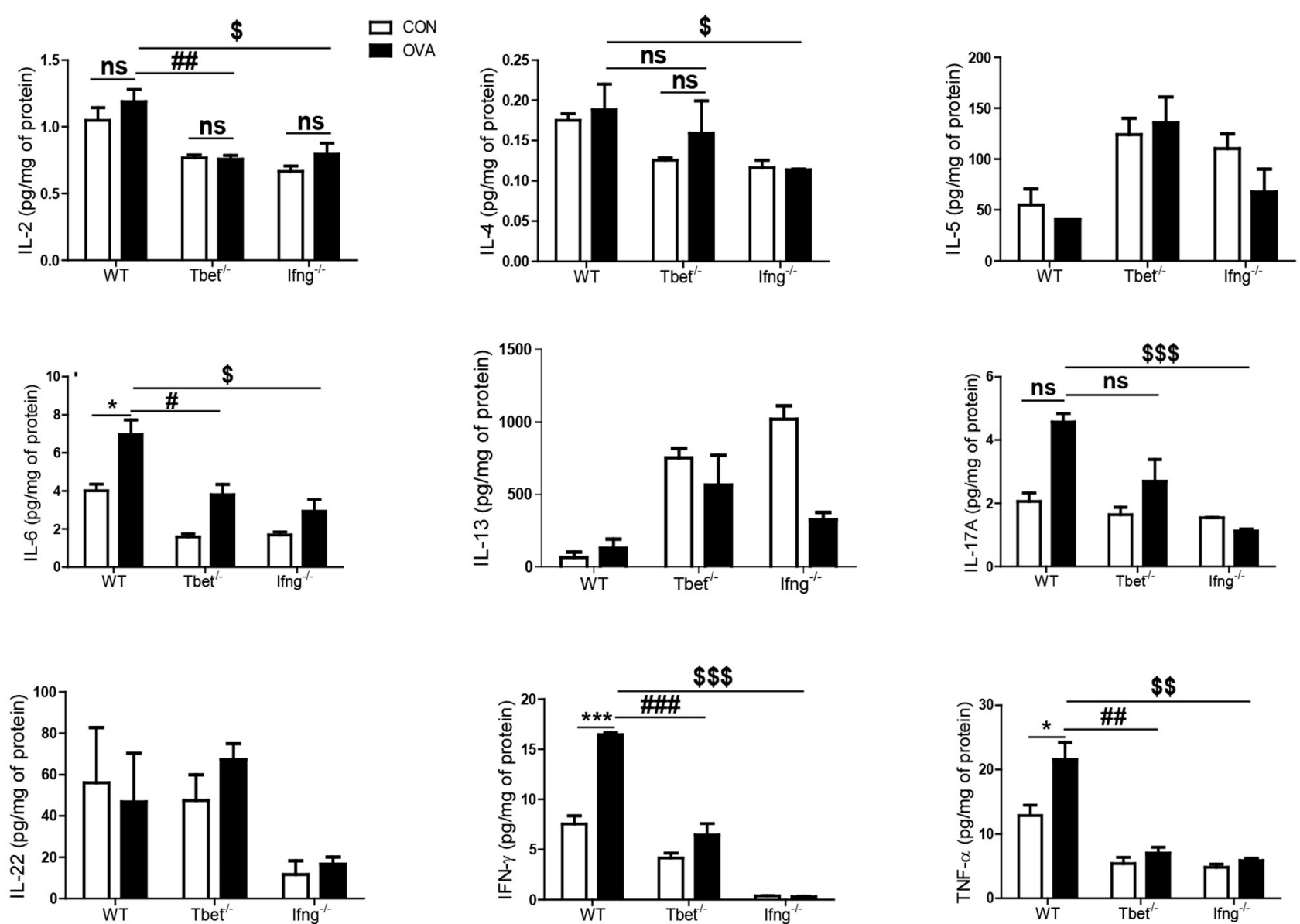

FIGURE 3 | Cytokines alteration by intratracheal OVA exposure in colon of WT, Tbet ${ }^{-/-}$, and Ifng ${ }^{-/-}$mice. The levels of IL-2, IL-4, IL-5, IL-6, IL-13, IL-17A, IL-22, IFN- $\gamma$, and TNF- $\alpha$ were measured in the colonic homogenates from WT $(n=16)$, Tbet ${ }^{-/-}(n=15)$ and Ifng ${ }^{-/-}(n=16)$ mice. Data are presented as the mean \pm SEM and $p$-value was estimated by unpaired $t$-test ${ }^{\star} P<0.05,{ }^{\star \star \star} P<0.001, \# P<0.05, \# \# P<0.01, \# \# \# P<0.001, \$ P<0.05, \$ \$ P<0.01, \$ \$ \$ P<0.001$, and ns, not significant).

\section{FTY720 Treatment Inhibits Colonic Inflammation Induced by Chronic Intratracheal OVA}

Researchers are currently trying to identify the mechanisms of interaction between the gastrointestinal and respiratory systems (22). We hypothesized that circulating immune cells could mediate cross-talk between the lungs and colon, specifically, that chronic intratracheal OVA-induced colitis could be mediated by circulating activated CD4 T cells. Activated CD4 T cells in the intrathoracic lymph nodes can migrate to other organs and cause a pathological response. To determine whether our hypothesis was correct, we first measured $\alpha 4 \beta 7$ integrin expression in circulating $\mathrm{T}$ cells. $\alpha 4 \beta 7$ is important in the homing of $\mathrm{T}$ cells to intestinal sites and is required for the induction of chronic colitis (23). The level of $\alpha 4 \beta 7$-expressing CD4 T cells was increased by chronic intratracheal OVA exposure in the blood and spleen, indicating that these OVA-stimulated cells may migrate to the colon and cause inflammation (Figure 1E). In addition, i.p. treatment with the immunomodulator FTY720, which interferes with the trafficking of cells between lymphoid organs and the blood (24) and causes lymphopenia (25) inhibited the progress of colitis and increased the colon weight/length ratio (Figure 1B), but did not improve lung inflammation (Figure 1F). This was accompanied by decreased $\alpha 4 \beta 7$ expression in CD $4 \mathrm{~T}$ cells (Figure 1E). These data suggest that circulating immune cells mediate chronic intratracheal OVA-induced colitis. FTY720 treatment also led to lymphopenia and affected both the percentage of total lymphocytes $(69.98 \pm 1.182 \%$ to $44.77 \pm$ 1.320, $P<0.001)$ and CD4 cells $(26.40 \pm 1.895 \%$ to $13.70 \pm$ $0.6234, P<0.001)$ in the spleen.

\section{T-Bet and IFN- $\gamma$ Are Crucial for Chronic Intratracheal OVA Exposure Induced Colitis}

To confirm that induction of colitis after chronic OVA exposure is mediated via Th1 responses, we challenged $\mathrm{Tbet}^{-/-}$and Ifng $^{-/-}$mice with OVA. Chronic OVA exposure did not alter colon length in Tbet $^{-/-}$or Ifng ${ }^{-/-}$mice (Figures 2A,B). Histological analysis confirmed that chronic OVA exposure 

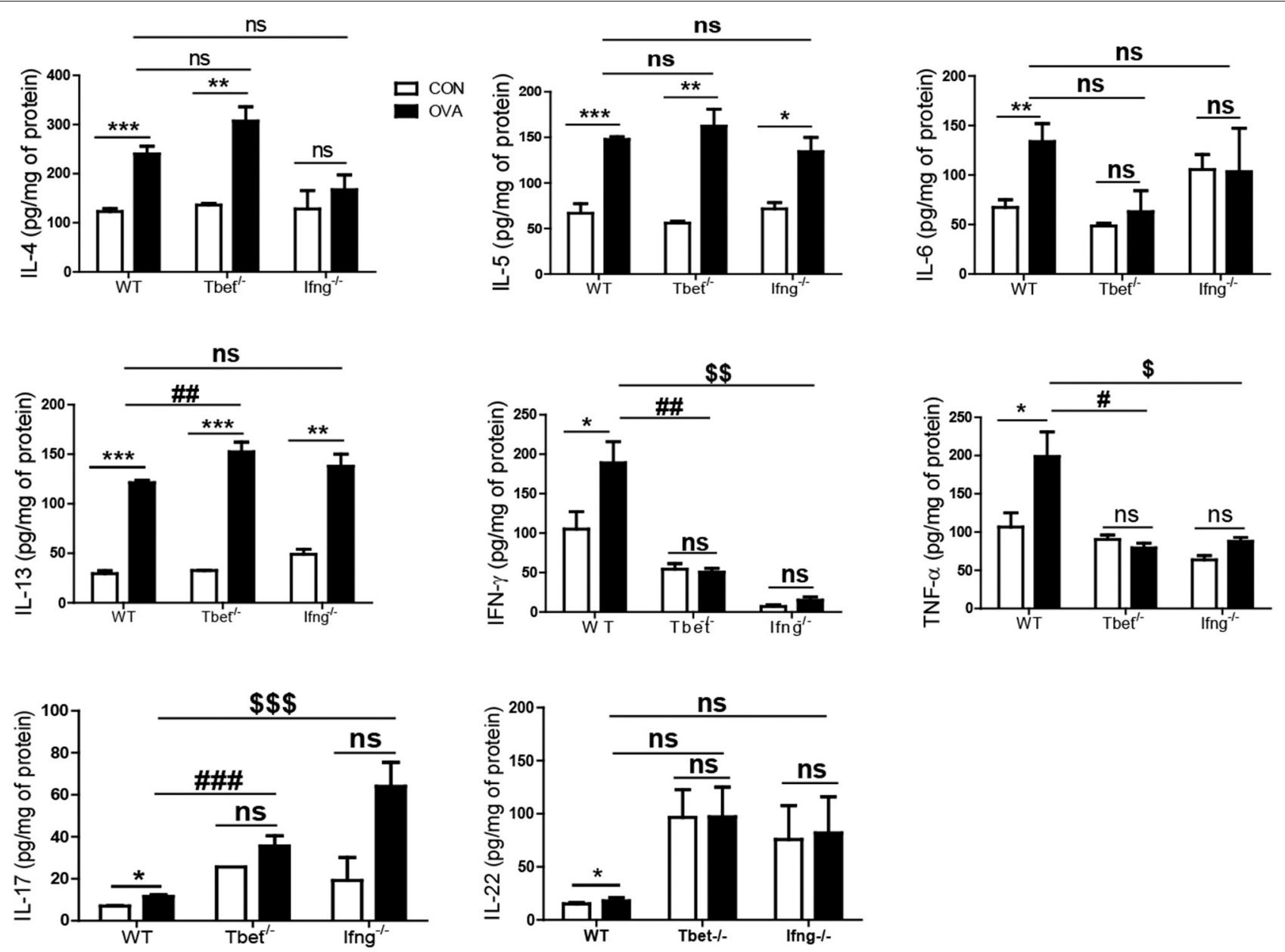

FIGURE 4 | Cytokines alteration by intratracheal OVA exposure in the lung of WT, Tbet ${ }^{-/-}$, and Ifng ${ }^{-/-}$mice. The levels of IL-4, IL-5, IL-6, IL-13, IL-17A, IL-22, IFN- $\gamma$, and TNF- $\alpha$ were measured in the lung homogenates from WT $(n=16)$, Tbet ${ }^{-/-}(n=15)$ and Ifng ${ }^{-/-}(n=16)$ mice. Data are presented as the mean \pm SEM and $p$-value was estimated by unpaired $t$-test $\left({ }^{\star} P<0.05\right.$, ${ }^{\star \star} P<0.01,{ }^{\star \star \star} P<0.001, \# P<0.05$, $\# \# P<0.01$, $\# \# \# P<0.001$ WT OVA vs. Tbet $-/-0$ OVA, $\$ P<$ $0.05, \$ \$ P<0.01, \$ \$ \$ P<0.001$ WT OVA vs. Ifng $-/-$ OVA, and ns, not significant).

induces colitis mediated through Th1 responses. OVA-challenged WT mice exhibited severe inflammation and damage in the colon. Colonic tissue from OVA-exposed mice showed thickened muscularis and increased inflammatory cell infiltration into the mucosa, whereas OVA challenge in $\mathrm{Tbet}^{-/-}$and $\mathrm{Ifng}^{-/-}$ mice did not result in histological damage (Figures 2C,D). Additionally, T-bet or IFN- $\gamma$ deficiency restored the OVAinduced overproduction of inflammatory cytokines, including TNF- $\alpha$, IL-6, and INF- $\gamma$ (Figure 3).

\section{T-Bet and IFN- $\gamma$ Are not Critical for OVA Exposure Induced Allergic Airway Inflammation}

It is well-known that the allergic airway inflammation is dependent on Th2 responses and independent of Th1 responses (26). Considering that development of colitis after chronic exposure to OVA was dependent on Th1 responses, we examined if Th1 responses play a role in OVA-induced allergic airway inflammation. As shown Figure 4, OVA exposure significantly increased the production of Th1, Th2, and Th17 cytokines in the lungs of WT mice compared with the control group.

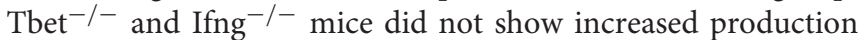
of Th1 or Th17 cytokines (IFN- $\gamma$, TNF- $\alpha$, IL-6, IL-17, and IL22 ), however they showed significantly increased production of Th2 cytokines (IL-4, IL-5, and IL-13 in Tbet $^{-/-}$mice; IL-5 and IL-13 in Ifng ${ }^{-/}$mice). Notably, our data showed that expressions of IFN- $\gamma$, TNF- $\alpha$ and IL- 6 were significantly increased in the lung of OVA-challenged WT mice. Tbet ${ }^{-/-}$ and Ifng ${ }^{-/-}$mice did exhibit alterations in Th1 response, as expected. Altogether, the data suggest that OVA exposure leads to Th1, Th2, and Th17 responses in the lungs of C57BL/6J mice and that Th1 responses are not critical for OVA-induced allergic airway inflammation. In addition, OVA exposure resulted in the significant infiltration of inflammatory cells into the lung parenchyma in all WT, Tbet $^{-/-}$, and Ifng $^{-/-}$mice. 

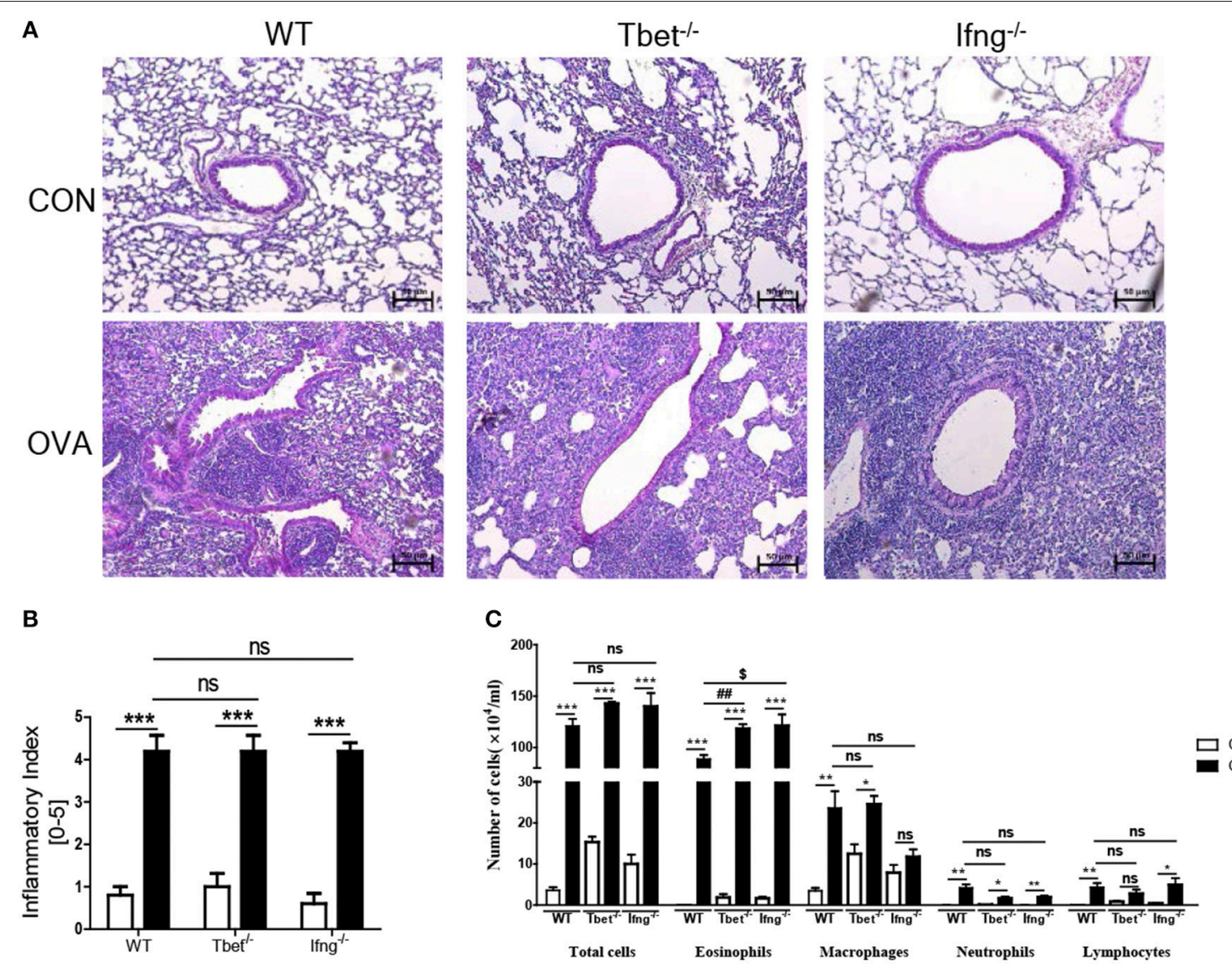

FIGURE 5 | Pathological changes in the lung after intratracheal OVA exposure. (A) Representative image of lung histology. (B) Assessment of histological changes of severity of allergic airway inflammation was quantified using a scoring system. (C) Inflammatory cell profiles in the BAL fluid after OVA exposure. WT ( $n=16)$, Tbet ${ }^{-/-}$ $(n=15)$ and lfng ${ }^{-/-}(n=16)$ mice. Data are presented as the mean \pm SEM and $p$-value was estimated by unpaired $t$-test ${ }^{\star} P<0.05$, ${ }^{\star \star} P<0.01,{ }^{\star \star \star} P<0.001$, $\# \# P<0.01, \$ P<0.05$, and ns, not significant).

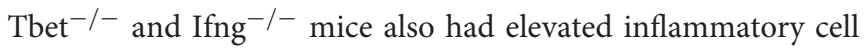
accumulation around the bronchi following OVA challenge, similar to the observations made in WT mice (Figure 5). Therefore, these data suggest that OVA-induced allergic airway inflammation is not dependent on Th1 responses, as opposed to OVA-induced colitis.

\section{DISCUSSION}

Recently, the link between colitis and pulmonary diseases has been discussed in the literature. Several cross-sectional studies reported an increased risk of colitis in patients with allergic disorders $(12,22,27)$. While it is well-known that pathogen exposure of the lungs leads to development of allergic responses in the lung, the effects on a remote site such as in the colon still remained uncertain (22). We demonstrated that cigarette smoke induced colitis via IFN- $\gamma$ (28) whilst another group showed that impaired gas exchange associated with cigarette smoke caused systemic and intestinal ischemia, driving angiogenesis and gastrointestinal tract epithelial barrier dysfunction, and resulting in the increased risk and severity of Crohn's disease (29). This study confirmed that colitis is mediated by the Th1 response, in particular IFN- $\gamma$-producing CD4 T cells. Based on our previous study, we confirmed the induction of colitis by exposing the airway to an allergen (OVA) and clarified the mechanism of this induction.

Our experiments demonstrate that the Th1 cell-specific transcription factor T-bet is robustly involved in OVA-induced colitis. Intratracheal OVA-induced colitis resulted in production of T-bet mediated cytokines, including TNF- $\alpha$, IL-6, and IFN- $\gamma$ in normal mice, while OVA exposure of T-bet deficient mice failed to result in induction of colitis. Furthermore, experimentation using IFN- $\gamma$ deficient mice confirmed that IFN- $\gamma$ mediates airway allergic response induced colitis. In fact, our results support the general notion that pathogenesis of Crohn's disease is predominantly associated with a Th1 response, but not with Th2 response (30). As IFN- $\gamma$ plays a necessary role in the development of colitis (31), anti-IFN$\gamma$ has been applied as a targeted therapy in the treatment of Crohn's disease (32). Recently, involvement of the Th17 response in the pathogenesis of colitis has been reported 
(33). In our experiment, intratracheal administration of OVA caused a stronger Th17 response in the lungs of T-bet deficient mice than in WT mice. This agrees with previous studies, which have demonstrated that T-bet suppresses Th17 differentiation, consequently leading to higher levels of IL$17 \mathrm{~A}$ in T-bet $\mathrm{KO}$ mice than in WT mice (34). However, intratracheal administration of OVA did not alter IL-17A levels in the colons of WT, T-bet deficient, or IFN- $\gamma$ deficient mice. The IFN- $\gamma$ pathway is not the only mechanism linking inflammatory bowel disease and pulmonary inflammation; a recent study demonstrated the role of macrophages in cigarette smoke-induced colitis (35). Indeed, we also observed an increase in the number of macrophages in the colonic laminar propria after OVA sensitization (Supplementary Figure 1), thus detailed mechanisms need to be identified in subsequent studies.

FTY720 treatment can influence the development of asthma, however the effects may differ depending on the mode of administration. Sawicka et al. used the adoptive transfer model for Th1 and Th2 cells in asthma development. In their model, FTY720 inhibited the migration of Th1 and Th2 cells to the lungs, resulting in attenuated asthma as FTY720 also prevents the migration of lymphocytes to inflammatory sites (13). Idzko et al. applied FTY720 locally via inhalation, which inhibited the migration of lung DCs to the mediastinal lymph nodes and blocked the formation of Th2 cells in the lymph nodes (36). In our experiments, we i.p. injected FTY720 to block the migration of inflammatory cells to the colon. Systemic FTY720 treatment may have less of an effect on the primary site of inflammation than local treatment, and may explain why our FTY720 treatment did not affect the development of asthma.

\section{REFERENCES}

1. Neurath MF. Cytokines in inflammatory bowel disease. Nat Rev Immunol. (2014) 14:329-42. doi: 10.1038/nri3661

2. Strober W, Zhang F, Kitani A, Fuss I, Fichtner-Feigl S. Proinflammatory cytokines underlying the inflammation of Crohn's disease. Curr Opin Gastroenterol. (2010) 26:310-7. doi: 10.1097/MOG.0b013e328339d099

3. Schroder K, Hertzog PJ, Ravasi T, Hume DA. Interferon-gamma: an overview of signals, mechanisms and functions. J Leukoc Biol. (2004) 75:163-89. doi: 10.1189/jlb.0603252

4. Bouma G, Strober W. The immunological and genetic basis of inflammatory bowel disease. Nat Rev Immunol. (2003) 3:521-33. doi: 10.1038/nri1132

5. Katakai T, Hara T, Sugai M, Gonda H, Shimizu A. Th1-biased tertiary lymphoid tissue supported by CXC chemokine ligand 13-producing stromal network in chronic lesions of autoimmune gastritis. J Immunol. (2003) 171:4359-68. doi: 10.4049/jimmunol.171.8.4359

6. Lundin KE, Nilsen EM, Scott HG, Loberg EM, Gjoen A, Bratlie J, et al. Oats induced villous atrophy in coeliac disease. Gut. (2003) 52:1649-52. doi: 10.1136/gut.52.11.1649

7. Wang Z, Zheng T, Zhu Z, Homer RJ, Riese RJ, Chapman HA Jr, et al. Interferon gamma induction of pulmonary emphysema in the adult murine lung. J Exp Med. (2000) 192:1587-600. doi: 10.1084/jem.192.11.1587

8. Fenta YA, Tello N, Jung JA, Urm SH, Loftus EV Jr, Yawn BP, et al. Inflammatory bowel disease and asthma: a population-based, case-control study. Inflamm Bowel Dis. (2010) 16:1957-62. doi: 10.1002/ibd.21277
This initial study indicates a promising future of potential therapeutic discovery and subsequent treatment of colitis in patients with allergic airway inflammation, including asthma. We found a circulating CD4 T cell mediated mechanism for interaction between the gastrointestinal and respiratory systems. It, however is still remained a lack of understanding of the cross-talk between lung and colon. Further studies are needed to broaden our knowledge of the cross-talk between lung and colon.

\section{AUTHOR CONTRIBUTIONS}

$\mathrm{HB}$ and $\mathrm{JK}$ conceived and designed the experiments. K-HJ and DS performed the majority of experiments. WK, DM, and SK contributed to the acquisition of data. GL wrote the paper. All authors read and approved the final manuscript.

\section{FUNDING}

This work was supported by a National Research Foundation of Korea (NRF) grant funded by the Korean government (MSIP) (NRF-2017R1A2B3009574).

\section{SUPPLEMENTARY MATERIAL}

The Supplementary Material for this article can be found online at: https://www.frontiersin.org/articles/10.3389/fimmu. 2019.00530/full\#supplementary-material

Supplementary Figure 1 | Colonic lamina propria and spleen were isolated and single cell suspensions prepared. After incubation with CD45-FITC, CD11b-PE, and F4/80-APC antibodies, the cells were analyzed using flow cytometry. Upper panel shows the percentage of macrophages in colonic lamina propria and the lower panel macrophages in spleen.
9. Keely S, Talley NJ, Hansbro PM. Pulmonary-intestinal cross-talk in mucosal inflammatory disease. Mucosal Immunol. (2012) 5:7-18. doi: $10.1038 / \mathrm{mi} .2011 .55$

10. Rutten EPA, Lenaerts K, Buurman WA, Wouters EFM. Disturbed intestinal integrity in patients with COPD: effects of activities of daily living. Chest. (2014) 145:245-52. doi: 10.1378/chest.13-0584

11. Peng YH, Liao WC, Su CH, Chen HJ, Hsia TC, Chu CC, et al. Association of inflammatory bowel disease with asthma risk: a nationwide cohort study. Allergy Asthma Proc. (2015) 36:e92-98. doi: 10.2500/aap.2015.36.3869

12. Brassard P, Vutcovici M, Ernst P, Patenaude V, Sewitch M, Suissa $\mathrm{S}$, et al. Increased incidence of inflammatory bowel disease in Quebec residents with airway diseases. Eur Respir J. (2015) 45:962-8. doi: 10.1183/09031936.00079414

13. Sawicka E, Zuany-Amorim C, Manlius C, Trifilieff A, Brinkmann V, Kemeny $\mathrm{DM}$, et al. Inhibition of Th1- and Th2-mediated airway inflammation by the sphingosine 1-phosphate receptor agonist FTY720. J Immunol. (2003) 171:6206-14. doi: 10.4049/jimmunol.171.11.6206

14. Park S, Baek H, Jung KH, Lee G, Lee H, Kang GH, et al. Bee venom phospholipase A2 suppresses allergic airway inflammation in an ovalbumininduced asthma model through the induction of regulatory T cells. Immun Inflamm Dis. (2015) 3:386-97. doi: 10.1002/iid3.76

15. Jung KH, Baek $H$, Shin D, Lee G, Park S, Lee S, et al. Protective effects of intratracheally-administered bee venom phospholipase A2 on ovalbumin-induced allergic asthma in mice. Toxins. (2016) 8:E269. doi: $10.3390 /$ toxins 8100269 
16. Lee EJ, Lee G, Sohn SH, Bae H. Extract of rhus verniciflua stokes enhances Th1 response and NK cell activity. Mol Cell Toxicol. (2016) 12:399-407. doi: 10.1007/s13273-016-0044-8

17. Choi SJ, Lee SH, Lee SJ, Yang MJ, Lee K. Subchronic inhalation toxicity study of 3R4F reference cigarette smoke in rats. Mol Cell Toxicol. (2016) 12:313-25. doi: 10.1007/s13273-016-0036-8

18. Erben U, Loddenkemper C, Doerfel K, Spieckermann S, Haller D, Heimesaat MM, et al. A guide to histomorphological evaluation of intestinal inflammation in mouse models. Int J Clin Exp Pathol. (2014) 7:4557-76.

19. Tate MD, Deng YM, Jones JE, Anderson GP, Brooks AG, Reading PC. Neutrophils ameliorate lung injury and the development of severe disease during influenza infection. J Immunol. (2009) 183:7441-50. doi: 10.4049/jimmunol.0902497

20. Ruane D, Brane L, Reis BS, Cheong C, Poles J, Do Y, et al. Lung dendritic cells induce migration of protective T cells to the gastrointestinal tract. J Exp Med. (2013) 210:1871-88. doi: 10.1084/jem.20122762

21. Nair PM, Starkey MR, Haw TJ, Liu G, Horvat JC, Morris JC, et al. Targeting PP2A and proteasome activity ameliorates features of allergic airway disease in mice. Allergy. (2017) 72:1891-903. doi: 10.1111/all.13212

22. Tulic MK, Piche T, Verhasselt V. Lung-gut cross-talk: evidence, mechanisms and implications for the mucosal inflammatory diseases. Clin Exp Allergy. (2016) 46:519-28. doi: 10.1111/cea.12723

23. Kurmaeva E, Lord JD, Zhang S, Bao JR, Kevil CG, Grisham MB, et al. $\mathrm{T}$ cell-associated alpha4beta7 but not alpha4betal integrin is required for the induction and perpetuation of chronic colitis. Mucosal Immunol. (2014) 7:1354-65. doi: 10.1038/mi.2014.22

24. Brinkmann V, Cyster JG, Hla T. FTY720: sphingosine 1-phosphate receptor1 in the control of lymphocyte egress and endothelial barrier function. Am J Transplant. (2004) 4:1019-25. doi: 10.1111/j.1600-6143.2004.00476.x

25. Morris MA, Gibb DR, Picard F, Brinkmann V, Straume M, Ley K. Transient $\mathrm{T}$ cell accumulation in lymph nodes and sustained lymphopenia in mice treated with FTY720. Eur J Immunol. (2005) 35:3570-80. doi: 10.1002/eji.2005 26218

26. Murdoch JR, Lloyd CM. Chronic inflammation and asthma. Mutat Res. (2010) 690:24-39. doi: 10.1016/j.mrfmmm.2009.09.005

27. Cohen R, Robinson D Jr, Paramore C, Fraeman K, Renahan K, Bala M. Autoimmune disease concomitance among inflammatory bowel disease patients in the United States, 2001-2002. Inflamm Bowel Dis. (2008) 14:738-43. doi: 10.1002/ibd.20406

28. Lee G, Jung KH, Shin D, Lee C, Kim W, Lee S, et al. Cigarette smoking triggers colitis by IFN-gamma(+) CD4(+) T cells. Front Immunol. (2017) 8:1344. doi: $10.3389 /$ fimmu.2017.01344
29. Fricker M, Goggins BJ, Mateer S, Jones B, Kim RY, Gellatly SL, et al. Chronic cigarette smoke exposure induces systemic hypoxia that drives intestinal dysfunction. JCI Insight. (2018) 3:94040. doi: 10.1172/jci.insight.94040

30. Niessner M, Volk BA. Altered Th1/Th2 cytokine profiles in the intestinal mucosa of patients with inflammatory bowel disease as assessed by quantitative reversed transcribed polymerase chain reaction (RT-PCR). Clin Exp Immunol. (1995) 101:428-35. doi: 10.1111/j.1365-2249.1995.tb03130.x

31. Ito $\mathrm{R}$, Shin-Ya $\mathrm{M}$, Kishida $\mathrm{T}$, Urano A, Takada $\mathrm{R}$, Sakagami J, et al. Interferon-gamma is causatively involved in experimental inflammatory bowel disease in mice. Clin Exp Immunol. (2006) 146:330-8. doi: 10.1111/j.1365-2249.2006.03214.x

32. Cui D, Huang G, Yang D, Huang B, An B. Efficacy and safety of interferongamma-targeted therapy in Crohn's disease: a systematic review and metaanalysis of randomized controlled trials. Clin Res Hepatol Gastroenterol. (2013) 37:507-13. doi: 10.1016/j.clinre.2012.12.004

33. Feng T, Qin H, Wang L, Benveniste EN, Elson CO, Cong Y. Th17 cells induce colitis and promote Th1 cell responses through IL-17 induction of innate IL-12 and IL-23 production. J Immunol. (2011) 186:6313-8. doi: 10.4049/jimmunol.1001454

34. Lazarevic V, Chen X, Shim JH, Hwang ES, Jang E, Bolm AN, et al. T-bet represses $\mathrm{T}(\mathrm{H}) 17$ differentiation by preventing Runx1-mediated activation of the gene encoding RORgammat. Nat Immunol. (2011) 12:96-104. doi: 10.1038/ni.1969

35. Lim D, Kim W, Lee C, Bae H, Kim J. Macrophage depletion protects against cigarette smoke-induced inflammatory response in the mouse colon and lung. Front Physiol. (2018) 9:47. doi: 10.3389/fphys.2018.00047

36. Idzko M, Hammad H, Van Nimwegen M, Kool M, Muller T, Soullie T, et al. Local application of FTY720 to the lung abrogates experimental asthma by altering dendritic cell function. J Clin Invest. (2006) 116:2935-44. doi: 10.1172/JCI28295

Conflict of Interest Statement: The authors declare that the research was conducted in the absence of any commercial or financial relationships that could be construed as a potential conflict of interest.

Copyright (c) 2019 Jung, Shin, Kim, Min, Kim, Kim, Lee and Bae. This is an openaccess article distributed under the terms of the Creative Commons Attribution License (CC BY). The use, distribution or reproduction in other forums is permitted, provided the original author(s) and the copyright owner(s) are credited and that the original publication in this journal is cited, in accordance with accepted academic practice. No use, distribution or reproduction is permitted which does not comply with these terms. 